\title{
A Study of Cardiovascular Manifestations in HIV Positive Patients and Their Association with the CD4 Cell Count
}

\author{
Charan Thej Reddy ${ }^{1}$, Ananthakrishna Barkur Shastry², Sharath P. Madhyastha ${ }^{3}$, \\ Ganesh V. Shetty ${ }^{4}$, Vasudeva Acharya ${ }^{5}$, Akhila Doddamani ${ }^{6}$ \\ 1,2,3,4,5 Department of Medicine, Kasturba Medical College, Manipal Academy of Higher Education, \\ Manipal, Karnataka, India. ${ }^{6}$ Department of Community Medicine, Kasturba Medical College, \\ Manipal Academy of Higher Education, Manipal, Karnataka, India.
}

\section{ABSTRACT}

\section{BACKGROUND}

Cardiovascular diseases in HIV positive patients are being increasingly detected in the developing countries and is likely to become even more prevalent as HIV infected individuals live longer. It is found that prevalence of cardiovascular disease is high in people with lower CD4 cell counts. The present study is therefore undertaken to identify the cardiovascular involvement in HIV and its relation with CD4 cell count.

\section{METHODS}

A cross sectional observational study was conducted in a tertiary care hospital, from 2015 to 2017 which included 500 HIV patients. All patients were assessed clinically and underwent electrocardiogram (ECG), 2D echocardiography, CD4 cell count along with routine investigations. The presence of cardiac involvement was determined based on electrocardiogram and echocardiography findings. An attempt was made to correlate various cardiac finding with CD4 cell count.

\section{RESULTS}

One third of study population (34 \%) was found to have cardiovascular manifestations. Chest radiograph revealed cardiomegaly in 66 out of 170 patients. ECG abnormalities were found in $72.94 \%$ while the most common echo findings were fractional shortening of $<30 \%$ found in $44.1 \%$ of the patients. These echocardiographic abnormalities are more in patients with CD4 count less than 50 / $\mu \mathrm{L}$. Majority (54.12\%) of the study population had a CD4 cell count of less than 50 / $\mu \mathrm{L}$.

\section{CONCLUSIONS}

In the present study, one third of HIV infected patients had cardiovascular manifestations. The most common clinical cardiac diagnosis in patients with HIV infection was heart failure followed by pulmonary hypertension. Reduced fractional shortening followed by reduced ejection fraction were the most common echocardiographic findings. Patients with low CD4 count have increased cardiovascular manifestations.

\section{KEY WORDS}

HIV, Cardiovascular Diseases, CD4 Count
Corresponding Author: Dr. Sharath Madhyastha P., Assistant Professor, Department of Medicine, Kasturba Medical College, Manipal Academy of Higher Education, Manipal - 576104, Karnataka, India.

E-mail:dr.sharathymc@gmail.com

DOI: $10.14260 /$ jemds $/ 2021 / 2$

How to Cite This Article: Reddy CT, Shastry AB, Madhyastha SP, et al. A study of cardiovascular manifestations in HIV positive patients and their association with the CD4 cell count. J Evolution Med Dent Sci 2021;10(01):7-12, DOI: 10.14260/jemds/2021/2

Submission 05-09-2020,

Peer Review 07-11-2020,

Acceptance 14-11-2020,

Published 04-01-2021.

Copyright @ 2021 Charan Thej Reddy et al. This is an open access article distributed under Creative Commons Attribution License [Attribution 4.0 International (CC BY 4.0)] 


\section{BACKGROUND}

Human immunodeficiency virus (HIV) is a global epidemic, with cases reported from virtually every country. In India as per 2015 HIV Estimation report, the adult (15-49 years) HIV prevalence was estimated at $0.26 \%$ and around 2.1 million people living with HIV (PLHIV) in India. ${ }^{1}$ Although this may seem low, India is third in the world in terms of greatest number of PLHIV. CD4 (cluster of differentiation) cell laboratory testing is considered as an important part in HIV care since this parameter is used in staging and prognosticating the disease. The normal CD4 count for most of the laboratories falls in a range of 800 to 1050 cells / $\mu \mathrm{L}$. A CD 4 count of $<200$ cells / $\mu \mathrm{L}$ indicates clinical stage of acquired immunodeficiency syndrome (AIDS). One of the important predictors of clinical outcome is the improvement in CD4 cell count in response to highly active antiretroviral therapy (HAART). ${ }^{2}$

The wide range of cardiac problems associated with HIV / AIDS has been documented in many autopsy studies in literature. The various abnormalities include myocarditis, dilated cardiomyopathy (DCM), pulmonary hypertension, infective endocarditis, pericardial diseases like effusion, malignancies like myocardial Kaposi's sarcoma and vascular occlusion. ${ }^{2}$

The cardiac problems in HIV positive patients are becoming increasingly detected in the developing countries. The prevalence of HIV related heart disease reported in the literature ranges between 28 and 73 percent. $^{3}$ In spite of this, cardiovascular involvement can be overlooked in HIV / AIDS patients, because symptoms of shortness of breath, tiredness, and poor exercise tolerance are often attributed to other illnesses related to HIV infection. Involvement of myocardium (myocarditis and DCM) is an important entity in HIV positive patients. Various factors responsible for this myocardial involvement are autoimmune response to HIV infection, direct invasion of myocardium by the virus, presence of opportunistic infection, therapy related (HAART), nutritional deficiencies and sustained immune suppression. ${ }^{3}$

Early and timely recognition of cardiovascular involvement in HIV infection is therefore essential to predict the outcome. The relation of CD4 cell count is also crucial, as it correlates with severity of cardiovascular involvement. ${ }^{4}$ The present study is therefore undertaken to identify presence of cardiovascular involvement in HIV infection and their association with CD4 cell count.

\section{METHODS}

The present study is a cross-sectional observational time bound study conducted in a tertiary care, teaching hospital in Southern India from September 2015 to May 2017. This study included five hundred patients, who attended the medical outpatient department or admitted to the wards of the hospital and were diagnosed cases of HIV. Approval from the institutional ethics committee (IEC 486 / 2015) was taken and written informed consent was obtained from all the participants.
Inclusion criteria was diagnosed cases of HIV, aged more than 18 years with two times enzyme-linked immunosorbent assay (ELISA) positivity or western blot positivity. Exclusion criteria was known cases of congenital heart diseases, hypertension, diabetes mellitus, coronary artery disease and rheumatic heart disease.

All patients were assessed clinically by thorough history and general physical and systemic examination. All patients underwent investigations like complete blood counts, kidney function tests, liver function tests, fasting lipid profile, chest $\mathrm{X}$ ray, ECG and 2D echocardiogram. All patients are HIV ELISA positive (double confirmed) and western blot test was done for few patients. CD4 counts were done for all patients using flow cytometer. All participants had undergone appropriate screening test for opportunistic infections.

M Mode and two-dimensional transthoracic echocardiography (2D ECHO) with colour flow Doppler assessment was done for all patients. The left atrial (LA) dimensions, left ventricular (LV) end systolic and end diastolic dimensions, right ventricular (RV) end diastolic dimensions and LV fractional shortening (FS) were obtained as per conventions of American Society of Echocardiography. LV volumes were measured and ejection fraction (EF) was calculated. The presence of regional wall motion abnormalities (RWMA), regurgitation of valves, pericardial effusion and vegetations for evidence of infective endocarditis were noted.

The presence of cardiovascular abnormalities in HIV infection was determined based on ECG and 2D ECHO findings. An attempt was made to correlate various cardiac finding with CD4 $\mathrm{T}$ cell count. Detailed drug therapy along with the duration was noted for all the patients in the study population.

\section{Statistical Analysis}

All the collected data was entered in Microsoft Excel sheet and then analysed using SPSS software (version 15.0, SPSS South Asia Bangalore). Qualitative data was presented as frequency and percentages and analysed using chi-square test or Fisher's exact test (in case of $2 \times 2$ contingency tables). Quantitative data was presented as mean and standard deviation (SD). Statistical significance was defined as $\mathrm{p}<0.05$.

\section{RESULTS}

Out of five hundred HIV patients 170 patients (34\%) had cardiac manifestations. The most common age group in the study population was $41-50$ years $(31.76 \%)$ with male preponderance $(57.05 \%)$. Around one third of the study population (35.9\%) belonged to the lower socioeconomic status group according to modified Kuppuswamy scale.

The most common cardiac symptoms among the study population was breathlessness $(46.47 \%)$ followed by palpitation (22.9\%), and chest pain (26.47\%). On examination, the common cardiac manifestations included tachycardia (25.88\%) followed by systolic murmur $(24.71 \%)$, basal crepitations (17.65 \%), pedal oedema (15.29\%), parasternal heave $(12.35 \%)$, and raised jugular venous pressure (10.59\%). The most common cardiac diagnosis among the study population was heart failure $(24.1 \%)$ 
followed by pulmonary arterial hypertension-PAH (12.4\%), dilated cardiomyopathy (7.6\%) and pericardial effusion with $\operatorname{rub}(5.29 \%)$.

\begin{tabular}{|ccc|}
\hline Examination Findings & Frequency Percentage \\
Pedal oedema & 26 & 15.29 \\
Tachycardia (Heart rate > 100 beats / min) & 44 & 25.88 \\
Raised Jugular venous pressure (JVP) & 18 & 10.59 \\
Parasternal Heave & 21 & 12.35 \\
Systolic murmur (Pan systolic in mitral and tricuspid & 42 & 24.71 \\
area and Ejection systolic murmur in pulmonary area) & 30 & 17.65 \\
Basal Crepitations & & \\
Diagnosis & 21 & 12.4 \\
Pulmonary Arterial Hypertension (PAH) & 41 & 24.1 \\
Heart failure & 13 & 7.6 \\
Dilated cardiomyopathy (DCM) & 9 & 5.29 \\
\hline Pericardial effusion with pericardial rub & \\
\hline Table 1. Cardiac Examination Findings and & \\
Diagnosis in the Study Population & \\
\hline
\end{tabular}

The haemoglobin of study population was on the lower side $(8.98 \pm 1.07 \mathrm{gm} / \mathrm{dl})$ with erythrocyte sedimentation rate (ESR) of $37.49 \pm 8.43$. Serum creatinine is slightly increased $(1.6 \pm 0.93)$ with normal blood urea nitrogen-BUN $(20.87 \pm$ 1.7). The fasting lipid profile is given in (Table 2).

\begin{tabular}{|ccc|}
\hline Lipid Profile Parameters & Mean & SD \\
Total Cholesterol & 162.72 & 30.89 \\
Triglycerides & 136.13 & 36.93 \\
HDL - C & 40.71 & 8.86 \\
LDL - C & 94.52 & 26.06 \\
VLDL - C & 27.49 & 8.43 \\
\hline Table 2. Lipid Profile in the Study Population & \\
with Cardiac Manifestations & \\
\hline
\end{tabular}

Majority (54.12\%) of the study population had a CD4 count of less than 50 cells / microliter.

The most common abnormal chest x-ray finding in the study population was cardiomegaly (38.82 \%) followed by pneumonia (12.94\%) and miliary pattern (4.76\%). The most common abnormal ECG findings amongst study population was sinus tachycardia (22.941\%) followed by right axis deviation (16.471\%) and low voltage complexes (15.294\%). The most common echo findings among the study population was fractional shortening of $<30 \%(44.1 \%)$, followed by reduced ejection fraction $(<50 \%)(24.1 \%)$ and pericardial effusions (22.9\%). (Table 3 )

\begin{tabular}{|ccc|}
\hline Chest X-Ray & Frequency & Percentage (\%) \\
\hline Normal cardiac shadow & 68 & 40.00 \\
Cardiomegaly & 66 & 38.82 \\
Pneumonia & 22 & 12.94 \\
Heart failure & 7 & 4.12 \\
Miliary pattern & 7 & 4.12 \\
ECG findings & & \\
Sinus tachycardia & 39 & 22.941 \\
Left axis deviation & 18 & 10.588 \\
Low voltage complexes & 26 & 15.294 \\
Right axis deviation & 28 & 16.471 \\
Intraventricular conduction delay & 13 & 7.647 \\
Echo findings & & \\
Reduced ejection fraction (<50 \%) & 41 & 24.1 \\
Fractional shortening (<30 \%) & 75 & 44.1 \\
\hline Pericardial effusions & 39 & 22.9 \\
PAH & 21 & 12.4 \\
Dilated cardiomyopathy & 13 & 7.6 \\
RWMA (anterior wall) & 3 & 1.8 \\
Diastolic dysfunction (a > e) & 15 & 8.8 \\
CD4 count & & \\
< 50 & 92 & 54.12 \\
50 - 200 & 51 & 30 \\
$>$ 200 & 27 \\
\hline Table 3. Chest X-Ray, ECG and 2D ECHO Findings \\
among the Study Population \\
\hline
\end{tabular}

The most common echo findings in patients with CD4 T cell count below $50 / \mu \mathrm{L}$ were fractional shortening $(<30 \%)$ in 41 patients, followed by reduced ejection fraction $(<50 \%)$ in 27 patients, and pericardial effusion in 21 patients, while in patients with CD4 count between 50 - 200 units, fractional shortening $(<30 \%)$ in 19 patients, followed by reduced EF $(<$ $50 \%$ ) in 9 patients and pericardial effusion in 10 patients. (Table 4)

\begin{tabular}{|ccccc|}
\hline Echo Findings & \multicolumn{5}{c}{ CD 4 Count } & Total \\
& $<\mathbf{5 0}$ & $\mathbf{5 0}-\mathbf{2 0 0}$ & $>\mathbf{2 0 0}$ & \\
Reduced ejection fraction $(<50 \%)$ & 27 & 9 & 5 & 41 \\
Fractional shortening $(<30 \%)$ & 41 & 19 & 15 & 75 \\
Pericardial effusions & 21 & 10 & 8 & 39 \\
PAH & 13 & 3 & 5 & 21 \\
Dilated cardiomyopathy & 7 & 4 & 2 & 13 \\
RWMA (anterior wall) & 1 & 1 & 1 & 3 \\
Diastolic dysfunction (a $>$ e) & 6 & 6 & 3 & 15 \\
\hline Table 4. Comparison of ECHO Findings with CD4 Count in the \\
Study Population with Cardiac Manifestations \\
\hline Chi square value - 15.859, df - 18, P value - 0.60 \\
\hline
\end{tabular}

In patients with CD4 count less than $50 / \mu \mathrm{L}$, mild, moderate and severe pulmonary hypertension was present in $8 \%, 31 \%$ and $62 \%$ of study population while in patients with CD4 count between 50 - 200, mild, moderate and severe pulmonary hypertension was present in $0 \%, 40 \%$ and $60 \%$ and in patients with CD4 count $>200$, mild, moderate and severe pulmonary hypertension was present in $67 \%, 33 \%$ and $0 \%$ of study population. (Table 5)

\begin{tabular}{|c|c|c|c|c|}
\hline \multirow[b]{2}{*}{ CD4 Count } & \multicolumn{3}{|c|}{ Pulmonary Hypertension } & \multirow[b]{2}{*}{$\begin{array}{l}\text { Total } \\
\text { N (\%) }\end{array}$} \\
\hline & $\begin{array}{l}\text { Mild } \\
\text { n (\%) }\end{array}$ & $\begin{array}{l}\text { Moderate } \\
\text { n (\%) }\end{array}$ & $\begin{array}{l}\text { Severe } \\
\text { n (\%) }\end{array}$ & \\
\hline Less than 50 & $1(8)$ & $4(31)$ & $8(62)$ & $13(100)$ \\
\hline $50-200$ & $0(0)$ & $2(40)$ & $3(60)$ & $5(100)$ \\
\hline$>200$ & $2(67)$ & $1(33)$ & $0(0)$ & $3(100)$ \\
\hline Total & $3(14)$ & 7 (33) & $11(52)$ & $21(100)$ \\
\hline \multicolumn{5}{|c|}{$\begin{array}{l}\text { Table 5. Comparison of Pulmonary Hypertension vs. CD4 Count } \\
\text { amongst the Study Population with Cardiac Manifestations }\end{array}$} \\
\hline hi square value - 7 & 2, $\mathrm{P}$ va & .001 & & \\
\hline
\end{tabular}

Most common antiretroviral therapy (ART) regimen used amongst study population was zidovudine (AZT) + lamivudine $(3 \mathrm{TC})+$ nevirapine (NVP) $(67.65 \%)$ followed by tenofovir (TDF) + emtricitabine (FTC) + efavirenz (EFV) (32.35\%) and stavudine $(\mathrm{d} 4 \mathrm{~T})+$ lamivudine $(3 \mathrm{TC})+$ nevirapine (NVP) $(5.88$ \%). (Table 6)

\begin{tabular}{|ccc|}
\hline Type of Therapy & Frequency & Percent \\
Zidovudine (AZT) + Lamivudine (3TC) + Nevirapine (NVP) & 115 & 67.65 \\
Tenofovir (TDF) + Emtricitabine (FTC) + Efavirenz (EFV) & 55 & 32.35 \\
Stavudine (D4T) + Lamivudine (3TC) + Nevirapine (NVP) & 10 & 5.88 \\
Total & $\mathbf{1 7 0}$ & $\mathbf{1 0 0}$ \\
\hline \multicolumn{3}{|c|}{ Table 6. Type of Therapy amongst Study Population } \\
with Cardiac Manifestations \\
\hline
\end{tabular}

Reduced ejection fraction $(<50 \%)$ was most common in more than 6 years in 30 patients followed by 3 to 6 years in 6 patients. Fractional shortening $(<30 \%)$ was most common in more than 6 years in 43 patients followed by 3 to 6 years in 21 patients. Pericardial effusions was most common in more than 6 years in 23 patients followed by 3 to 6 years in 6 patients. There was no statistically significant difference between echo findings and duration of therapy amongst study population. (Table 7). 


\begin{tabular}{|c|c|c|c|c|c|}
\hline \multirow[b]{2}{*}{ Echo Findings } & \multicolumn{4}{|c|}{ Duration of Therapy } & \multirow[b]{2}{*}{ Total } \\
\hline & $\begin{array}{l}<1 \text { Year } \\
\text { (n) }\end{array}$ & $\begin{array}{c}1 \text { to } 3 \\
\text { Years } \\
\text { (n) }\end{array}$ & $\begin{array}{l}3 \text { to } 6 \\
\text { Years }(n)\end{array}$ & $\begin{array}{c}\text { More than } 6 \\
\text { Years (n) }\end{array}$ & \\
\hline $\begin{array}{l}\text { Reduced ejection fraction } \\
\qquad<50 \%)\end{array}$ & 2 & 3 & 6 & 30 & 41 \\
\hline $\begin{array}{c}\text { Fractional shortening (< } \\
30 \%)\end{array}$ & 2 & 9 & 21 & 43 & 75 \\
\hline Pericardial effusions & 4 & 6 & 6 & 23 & 39 \\
\hline PAH & 2 & 3 & 3 & 13 & 21 \\
\hline Dilated cardiomyopathy & 1 & 1 & 4 & 7 & 13 \\
\hline RWMA (anterior wall) & 1 & 0 & 1 & 1 & 3 \\
\hline $\begin{array}{l}\text { Diastolic dysfunction (a > } \\
\text { e) }\end{array}$ & 3 & 2 & 4 & 6 & 15 \\
\hline \multicolumn{6}{|c|}{$\begin{array}{l}\text { Table 7. Comparison of ECHO Findings with Duration of Therapy } \\
\text { amongst Study Population with Cardiac Manifestations }\end{array}$} \\
\hline 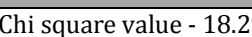 & 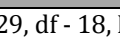 & & & & \\
\hline
\end{tabular}

\section{DISCUSSION}

In the present study, out of 500 patients diagnosed to have HIV / AIDS infection, 170 (34\%) had cardiac manifestations. The prevalence of cardiovascular abnormality in AIDS patients have been reported in the range of $28 \%$ to $73 \%$. $^{3}$

In the present study, the most common age group with cardiovascular manifestations was 41 - 50 years (31.76\%) with male predominance (57.05\%). Similarly, in the study conducted by Harminder Singh et al., the most common age group was that between 30 and 39 years, with more males affected than females. Because young male are the most exposed people due to forced migration, due to unemployment and exposure to commercial sex workers. ${ }^{5}$

In the present study, according to Kuppuswamy's scale, that most of the study population with cardiac manifestations belong to lower socioeconomic (SES) group (35.88\%). Similarly, in the study conducted by Harminder Singh et al., in which they reported that most of the patients belonged to the lower socioeconomic status (79.56\%) whereas, only $1.45 \%$ belong to high socioeconomic status. Lower socioeconomic status people in their study had sexual practices that differ from those of mainstream cultures. The early age of marriage, unemployment, unprotected sex and exposure to commercial sex workers contributed to the unchecked HIV spread. Low socio-economic status patients with limited educational and economic infrastructure, poor access to medicine and health facility availability end up in late diagnosis with lower CD4 count with increased cardiac manifestations. ${ }^{5}$

Among the cardiac symptoms, breathlessness (46.47\%) was most common, followed by chest pain (26.47\%) and palpitation (22.94\%). These findings are in correlation with the Mehul Marwadi study in which breathlessness was $33 \%$ and palpitation was 11 percent. 6

Most common clinical findings amongst study population was left heart failure $(24.1 \%)$ followed by pulmonary arterial hypertension (PAH) (12.4\%), dilated cardiomegaly (7.6\%) and valvular disease (5.29\%). Left heart failure and cardiomegaly are due to direct invasion of cardiac muscle by the HIV virus with or without myocarditis, bigger left ventricular mass (LV) in HIV infected patients, toxic effects of antiretroviral therapy (ART), concomitant opportunistic infections, as well as nutritional deficiencies. PAH is due to obliteration of pulmonary vasculature with medial hypertrophy and increased proliferation of endothelial and smooth muscle cells. ${ }^{7}$
The present study shows there is no alteration in the lipid profile; may be because patients were already on statins. The treatment of HIV, highly active antiretroviral therapy (HAART) has an adverse effect on lipid profile. It is also important to remember that HIV / AIDS infection itself can causes lipid abnormality especially elevated triglycerides and low highdensity lipoprotein cholesterol (HDL) levels as evident from pre-HAART era literatures. It was also observed that lower the CD4 $\mathrm{T}$ cell count, more unfavourable will be the lipid profile (hypertriglyceridemia and lower HDL-cholesterol levels). ${ }^{8}$

Most of the study population had CD4 T cell count below $50 / \mu \mathrm{L}(30 \%)$ followed by $50-199 / \mu \mathrm{L}(27.65 \%)$. This findings correlate well with the study conducted by Raman Kumar Sharma et al., in which out of 100 cases of HIV infected patients with cardiovascular manifestations, CD4 count was less than $50 / \mu \mathrm{L}$ in $27.3 \%, 34.6 \%$ of cases had CD4 count between 50 to $199 / \mu \mathrm{L}, 25.4 \%$ had CD4 Count 200 - 499 / $\mu \mathrm{L}$ while $12.7 \%$ had CD4 count $>500 / \mu$ L. ${ }^{9}$ Similarly, in the study conducted by Ayaskanta Singh et al., in which CD4 count was less than $50 / \mu \mathrm{L}$ in $28.6 \%, 32.9 \%$ of cases had CD4 count between 50 to 200 / $\mu \mathrm{L}$ while $25.7 \%$ had CD4 Count > 350 / $\mu \mathrm{L} .{ }^{10}$

In the present study, the most common ECG finding amongst HIV patients with cardiovascular manifestations was sinus tachycardia (22.9\%) followed by right axis deviation (16.4\%), low voltage complexes (15.294\%) and left axis deviation $(10.58 \%)$. These findings correlate well with the study conducted by Mehul Marwadi et al. in which out of 100 cases studied, sinus tachycardia was observed in $24 \%$ cases, left ventricular hypertrophy in $8 \%$ cases and low voltage complexes in $6 \%$ cases.

The sinus tachycardia might suggest early evidence of cardiac failure or left ventricular diastolic dysfunction. Isolated persistent tachycardia could be an early feature of myocarditis. Right axis deviation is because of pulmonary artery hypertension due to increased endothelin and platelet derived growth factor in HIV patients. ${ }^{11}$ Low voltage complexes were due to pericardial effusion and left axis deviation is due to increase in left ventricular mass. It is still uncertain as to why left ventricular mass increases in HIV positive patients. It is thought to be linked to mitochondrial toxicity. HIV viral particles can directly invade the myocardial tissue and cause cytokines release locally which in turn leads to inflammation, myocarditis and dilated cardiomyopathy. Left ventricular mass also linked to various other factors such as nutritional deficiency and presence of opportunistic infections. It was also found that a lower nadir CD4 cell count has been associated with higher left ventricular mass index. ${ }^{12,13}$ Meng et al.14 stated that posterior and interventricular septal wall thicknesses is more in patients who have received protease inhibitor (PI) compared to those who have not received.

In the present study, the most of common ECHO findings amongst study population was fractional shortening $(<30 \%)$ $(44.1 \%)$ followed by reduced ejection fraction $(<50 \%)(24.1$ $\%)$ and pericardial effusions (22.9\%). Fractional shortening represents degree of shortening of the LV diameter between end-diastole and end-systole, measure of contractility of the heart. Reduced fractional shortening is observed in early disease due to decreased contractility of the heart. Timely identification and treatment are crucial to avoid substantial morbidity from cardiac involvement. Similarly, in the study 
done by Mirri et al. ${ }^{15}$ prevalence of echocardiographic abnormalities was $17 \%$. Reduction in fractional shortening was the commonest manifestation found in their study (46.29 $\%)$. Reduced ejection fraction is due to direct HIV infection to the myocardium causing myocarditis and altered immunological response in HIV infected patients causing immunological damage to the heart. Dilated cardiomyopathy seen in HIV patients is due to HIV itself causing myocarditis and also because of activated multifunctional cytokines. The common reasons for pulmonary arterial hypertension in HIV positive patients are pulmonary infections, LV dysfunction and venous thromboembolism. ${ }^{16}$

In our study, the most of common ECHO findings in patients with CD4 count less than $50 / \mu \mathrm{L}$ was fractional shortening $(<30 \%)$ seen in 41 patients, followed by reduced ejection fraction $(<50 \%)$ in 27 patients and pericardial effusions in 21 patients while in patients with CD4 count between 50 - 199, fractional shortening $(<30 \%)$ in 19 patients, followed by reduced ejection fraction $(<50 \%)$ in 9 patients and pericardial effusions in 8 patients and this difference was statistically not significant. Previous studies done in India showed cardiac manifestations of HIV are frequently encountered in a state of profound immunosuppression with reduced CD4 T cell Count (CD4 < $200 / \mu \mathrm{L})^{10,17,18}$ The present study also revealed that HIV patients with CD4 count less than $200 / \mu \mathrm{L}$ had a higher prevalence of echocardiographic abnormalities than those with CD4 cell count more than $200 / \mu \mathrm{L}$. Level of immunosuppression is most significant factor in development of cardiac abnormalities and there is considerable correlation between CD4 $\mathrm{T}$ cell count and echocardiographic abnormalities. ${ }^{19}$ HIV infected patients with CD4+ lymphocyte counts less than $100 / \mu \mathrm{L}$ had more frequent abnormal echocardiograms than those with CD4+ lymphocytes counts > $100 / \mu \mathrm{L} .{ }^{20,21}$

In the present study, 12.4 percent patients had pulmonary artery hypertension. More number of severe pulmonary hypertension patients were found in CD4 count less than 50 / $\mu \mathrm{L}$ which was statistically significant. HIV is not known to infect endothelial cells and HIV associated proteins (gp120, Tat, Nef) may elevate pulmonary arterial pressure. Oxidative stress induced by these proteins may lead to endothelial dysfunction and vascular injury. ${ }^{22}$ Numerous host inflammatory cytokines like interleukin-6, tumour necrosis factor-alpha, interleukin-1 beta, and platelet derived growth factor, are increased in patients with HIV infection. These cytokines together can provoke a procoagulant state, upsurge the expression of endothelial adhesion molecules and increase the accumulation of inflammatory cells in the pulmonary arteries. ${ }^{23}$

In the present study, more number of patients are taking HIV drugs since six years or more. Even the echocardiographic abnormalities are more in those groups. It appears that longer the disease more will be the cardiac abnormalities but these differences were statistically not significant. With the advent of HAART, there is a significant raise in the number of people living with HIV infection. ${ }^{24}$ This increased life span of infected individuals has led to an increased incidence of cardiac abnormalities. About one half of HIV / AIDS cases reported some cardiovascular complications. These cardiovascular manifestations include diseases of myocardium (myocarditis, DCM), pulmonary arterial hypertension, pericardial effusion, infective endocarditis and coronary artery disease. Possible mechanisms of cardiac complications in HIV / AIDS comprise direct cardiotoxicity by the HIV itself, immune mediated, nutritional deficiencies and drug (HAART) induced. ${ }^{25,26}$

In the present study, the most common ART regimen used amongst study population was zidovudine (AZT) + lamivudine (3TC) + nevirapine (NVP) (67.5\%) followed by tenofovir (TDF) + emtricitabine (FTC) + efavirenz (EFV) (32.35\%) and stavudine (d4T) + lamivudine (3TC) + nevirapine (NVP) (5.88 $\%)$. These findings correlate well with the study conducted by Indumati $\mathrm{V}$ et al. 27 The main problems associated with ART are the HIV associated lipodystrophy syndrome and alterations in lipid and glucose metabolism. Also, a large number of patients may develop insulin resistance and hyperlipidaemia. Hence there is high concern that HIV infected patients treated with anti-retroviral therapy may be at increased risk of developing premature coronary artery diseases. ${ }^{28}$

Present study population was not on protease inhibitors but still had increased cardiovascular manifestations with duration of therapy probably due to ongoing viral inflammation caused by HIV in myocardium. HIV induced immune activation itself is considered to independently contribute to cardiovascular deaths and may partially explain the greater cardiovascular mortality amongst patients taking ART.29

\section{Limitations of the Study}

The number of naive patients with HIV included in the study were less. It needs further studies on effect of duration of therapy with different regimes on cardiovascular manifestations.

\section{CONCLUSIONS}

In the present study one third of HIV infected patients had cardiovascular manifestations with male preponderance. Most common clinical cardiac diagnosis in patients with HIV infection was heart failure followed by pulmonary artery hypertension. Reduced fractional shortening followed by reduced ejection fraction were the most common cardiovascular manifestations in HIV infected patients on echocardiogram. Patients with low CD4 count have increased cardiovascular manifestations. Increased prevalence of pericardial effusion and pulmonary artery hypertension were present in patients with lower CD4 count.

Data sharing statement provided by the authors is available with the full text of this article at jemds.com.

Financial or other competing interests: None.

Disclosure forms provided by the authors are available with the full text of this article at jemds.com.

\section{REFERENCES}

[1] National AIDS control organisation annual report 20112012. Department of AIDS control. 
[2] Malani PN. Harrison's principles of internal medicine. JAMA 2012;308(17):1813-4.

[3] Kaul S, Fishbein MC, Siegel RJ. Cardiac manifestations of acquired immune deficiency syndrome: a 1991 update. Am Heart J 1991;122(2):535-44.

[4] Gopal M, Bhaskaran A, Khalife WI, et al. Heart disease in patients with HIV / AIDS - an emerging clinical problem. Curr Cardiol Rev 2009;5(2):149-54.

[5] Singh H, Dulhani N, Bithika NK, et al. Rural epidemiology of HIV positive tribal patients from Chhattisgarh in India. Journal of Global Infectious Diseases 2010;2(1):39-42.

[6] Marwadi M, Doctor N, Gheewala G, et al. Cardiac manifestations in HIV / AIDS patients and their correlation with CD4 $+\mathrm{T}$ cell count. National Journal of Medical Research 2014;4(3):244-8.

[7] Prendergast BD. HIV and cardiovascular medicine. Heart 2003;89(7):793-800.

[8] Constans J, Pellegrin JL, Peuchant E, et al. Plasma lipids in HIV-infected patients: a prospective study in 95 patients. Eur J Clin Invest 1994;24(6):416-20.

[9] Kumar R, Vandegraaff N, Mundy L, et al. Evaluation of PCR - based methods for the quantitation of integrated HIV - 1 DNA. J Virol Methods 2002;105(2):233-46.

[10] Singh A, Das S, Dalai RK. Study of cardiac manifestations in patients with HIV infection and their correlation with CD4 count in Indian population. International Journal of Clinical Medicine 2012;3(3):178-83.

[11] Humbert M, Monti G, Fartoukh M, et al. Platelet - derived growth factor expression in primary pulmonary hypertension: comparison of HIV seropositive and HIV seronegative patients. Eur Respir J 1998;11(3):554-9.

[12] Lewis W. Cardiomyopathy in AIDS: a pathophysiological perspective. Prog Cardiovasc Dis 2000;43(2):151-70.

[13] Lewis W, Grupp IL, Grupp G, et al. Cardiac dysfunction occurs in the HIV-1 transgenic mouse treated with zidovudine. Lab Invest 2000;80(2):187-97.

[14] Meng Q, Lima JA, Lai H, et al. Use of HIV protease inhibitors is associated with left ventricular morphologic changes and diastolic dysfunction. J Acquir Immune Defic Syndr 2002;30(3):306-10.

[15] Mirri A, Rapezzi C, Iacopi F, et al. Cardiac involvement in HIV infection: a prospective, multicenter clinical and echocardiographic study. Cardiologia 1990;35(3):203-9.

[16] Barbaro G, Fisher SD, Pellicelli AM, et al. The expanding role of the cardiologist in the care of HIV infected patients. Heart 2001;86(4):365-7.
[17] Chang WT, Wu CC, Hung CC, et al. Left ventricular dysfunction is associated with CD4 lymphocyte count rather than opportunistic infection in human immunodeficiency virus infection. J Formos Med Assoc 2003;102(3):158-63.

[18] Kumar SKK, Jayakumar B, Baiju R. A study of clinical profile of cardiac dysfunction in patients with HIV infection. Int J Res Medi Sci 2016;4(12):5149-53.

[19] Barbaro G, Klatt EC. HIV infection and the cardiovascular system. Aids Rev 2002;4(2):93-103.

[20] Cardoso JS, Moura B, Martins L, et al. Left ventricular dysfunction in human immunodeficiency virus (HIV) infected patients. Int J Cardiol 1998;63(1):37-45.

[21] Herskowitz A, Willoughby SB, Baughman KL, et al. Cardiomyopathy associated with antiretroviral therapy in patients with HIV infection: a report of six cases. Ann Intern Med 1992;116(4):311-3.

[22] Mermis J, Gu H, Xue B, et al. Hypoxia - inducible factor - 1 $\alpha$ / platelet derived growth factor axis in HIV - associated pulmonary vascular remodeling. Respir Res 2011;12(1):103.

[23] Tcherakian C, Couderc LJ, Humbert M, et al. Inflammatory mechanisms in HIV - associated pulmonary arterial hypertension. Semin Respir Crit Care Med 2013;34(5):645-53.

[24] Boccara F. Cardiovascular complications and atherosclerotic manifestations in the HIV - infected population: type, incidence and associated risk factors. AIDS 2008;22(Suppl 3):S19-26.

[25] Mllei J, Grana D, Alonso GF, et al. Cardiac involvement in acquired immunodeficiency syndrome -- a review to push action. The committee for the study of cardiac involvement in AIDS. Clin Cardiol 1998;21(7):465-72.

[26] Aboud M, Elgalib A, Pomeroy L, et al. Cardiovascular risk evaluation and antiretroviral therapy effects in an HIV cohort: implications for clinical management: the CREATE 1 study. Int J Clin Pract 2010;64(9):1252-9.

[27] Indumati V, Vijay V, Rajeshwari AM, et al. Comparison of serum lipid profile in HIV positive patients on ART with ART naïve patients. J Clin Diagn Res 2014;8(10):CC06-9.

[28] Chow DC, Day LJ, Scott AS, et al. Metabolic complications of HIV therapy. IAPAC Pon 2006;12(9):302-17.

[29] Thienemann F, Sliwa K, Rockstroh JK. HIV and the heart: the impact of antiretroviral therapy: a global perspective. Eur Heart J 2013;34(46):3538-46. 